\title{
Ruptura de Vasos Prévios: Relato de Caso
}

\author{
Rupture of Vasa Previa: A Case Report \\ José Rafael Macéa, Maria Inez Marcondes Macéa \\ RESUMO
}

\begin{abstract}
No diagnóstico diferencial das hemorragias do terceiro trimestre da gestação, de causa exclusivamente obstétrica, incluem-se descolamento prematuro da placenta, inserção baixa da placenta (placenta prévia, com seus subtipos), ruptura uterina, ruptura do seio marginal placentário e ruptura de vasos prévios (VP). Os três primeiros diagnósticos são mais freqüentes, têm fatores epidemiológicos conhecidos e, portanto, são de diagnóstico mais fácil. Ocorre que há obstetras com larga experiência prática, assim como ultra-sonografistas especializados em Medicina Fetal, que, apesar de conhecerem o assunto em teoria, nunca se defrontaram na prática obstétrica com os VP e muito menos com sua ruptura. Motivou-nos escrever este artigo o fato de termos assistido e participado, após 32 anos de prática constante e ininterrupta de Obstetrícia, de forma dramática e pela primeira vez, de um caso de ruptura de VP que resultou em óbito fetal, durante o trabalho de parto. Os tratados de Obstetricia são muito concisos e repetitivos ao tratar do assunto. Porém, ao fazer uma revisão mais acurada sobre o assunto em tela, pudemos constatar que, apesar de tratar-se de eventualidade rara, uma fatalidade, há na literatura atual clara indicação de que é possivel o diagnóstico desta entidade mórbida durante a gestação, por meio de tecnologia mais avançada. Feito o diagnóstico, deve o tocólogo interromper a gestação, quando da maturidade fetal, e, deste modo, promover significativa diminuição das altas taxas de mortalidade fetal, que oscilam entre 33 e $100 \%$.
\end{abstract}

PALAVRAS-CHAVE: Vasa previa. Cordão umbilical: inserção velamentosa. Óbito fetal.

\section{Introdução}

A melhor definição de vasos prévios (VP), encontrada na literatura, pareceu-nos ser a de Pent ${ }^{1}$, que preceitua tratar-se de anomalia de inserção do funículo umbilical na placenta, na qual os vasos umbilicais cruzam o segmento inferior uterino, colocando-se à frente da apresentação.

$\mathrm{Na}$ imensa maioria das vezes a presença de VP coexiste com inserção velamentosa do funículo umbilical, situação na qual a gelatina de Wharton termina a distâncias variáveis da superfície placentária, fazendo com que os vasos umbilicais situem-se entre o âmnio e o córion, podendo ocorrer ou não os VP.

Wrisberg, em 1773, descreveu pela primeira vez a inserção velamentosa do funículo umbilical. Lobsten, em 1801, descreveu pela primeira os VP. Admite-se que entre 1801 e 1951 tenham sido descritos não mais que 100 casos de VP na literatura e não mais que 200 casos até $1979^{2}$.

Departamento de Morfologia da Faculdade de Ciências Médicas da Santa Casa de São Paulo

Correspondência: José Rafael Macéa

Avenida Angélica, 916 conj. 708

01228-000 - São Paulo - SP
A freqüência de inserção velamentosa do funículo umbilical oscila entre 0,5 e $1 \%{ }^{1,2}$ em gestações simples, ao redor de $10 \%$ nas gestações gemelares ${ }^{3,4}$ e, segundo alguns autores, pode chegar próximo de $100 \%$ em gestações trigemelares ${ }^{3,4}$.

A maior importância da ruptura dos VP reside no fato de que se relaciona com mortalidade fetal que pode chegar a $100 \%{ }^{5}$. Todavia, a simples compressão dos VP pela apresentação pode provocar mortalidade fetal entre 50 e $60 \%{ }^{5}$.

Eventualmente, um examinador mais atento poderá sentir ao toque vaginal um vaso fetal, sob a forma de estrutura tubular, revestido pelas membranas, pulsátil, em consonância com os batimentos cardíacos fetais. A compressão discreta deste vaso poderá provocar modificações imediatas na freqüência cardíaca fetal ${ }^{6}$.

O diagnóstico de VP é costumeiramente negligenciado em função da raridade desta entidade mórbida, da grande dificuldade diagnóstica e da escassez de tempo que decorre entre a suspeita diagnóstica e a intervenção médica, que deve ser imediata ${ }^{7}$.

Calcula-se ser a volemia de um feto a termo ao redor de $250 \mathrm{ml}^{7}$. Portanto, a perda de pequena quantidade de sangue fetal pode levá-lo ao choque hemorrágico e ao óbito. É preciso lembrar também 
que é possivel a ruptura dos VP com membranas integras ${ }^{8}$. Neste caso, a simples compressão dos vasos pela apresentação provocaria a ruptura dos mesmos. É necessário frisar que o sangramento vaginal nem sempre está presente ${ }^{8}$. Em muitas ocasiões, a ruptura dos VP com conseqüente sangramento vaginal pode ocorrer muitas horas após a ruptura das membranas, ou seja, à medida que evolui a dilatação cervical, aumenta o diâmetro da ruptura das membranas, o que em determinado momento englobará um vaso fetal, rompendo-o ${ }^{8}$.

Antes do advento da ultra-sonografia obstétrica, o diagnóstico de VP era casual e esporádico. Assim, por meio de um exame especular ou por amnioscopia poder-se-ia visualizar, de forma direta, um dos vasos cursando no interior das membranas fetais. Evidentemente, esses procedimentos citados para diagnóstico eventual dos VP carregavam o inconveniente de poder provocar a ruptura do vaso fetal e conseqüente hemorragia ${ }^{1}$.

Em muitos serviços dos Estados Unidos, todas as vezes que ocorrem perdas sangüineas vaginais na segunda metade gestacional, colhe-se este sangue e, mediante de métodos variados, pesquisa-se a presença de eritrócitos fetais ou de hemoglobina fetal. Quando esta pesquisa é positiva conclui-se que o sangue é de origem fetal e a possibilidade de VP é exaustivamente pesquisada ${ }^{2-6}$.

Apesar da utilização de todos os métodos acima descritos, a mortalidade perinatal por ruptura de VP, até o início dos anos 90, variava de 33 a $100 \%{ }^{3}$.

Recentemente com a utilização da ultrasonografia com escala de cinza e, principalmente, com a associação da ultra-sonografia endovaginal à dopplerfluxometria a cores, o diagnóstico pré-parto de VP tornou-se muito mais freqüente e seguro ${ }^{7,8}$.

\section{Relato do Caso}

Tercigesta, 40 anos, dois partos normais. A colheita de vilo corial na $11^{\text {a }}$ semana gestacional demonstrou cariótipo $46 \mathrm{XY}$. A ultra-sonografia da 18 a semana mostrou inserção baixa de placenta, que cobria o orificio interno da cérvice uterina. Ultra-sonografias repetidas realizadas em dois serviços demonstraram "migração" placentária para a face posterior alta da cavidade uterina. Foi submetida a oito ultra-sonografias no decorrer da gestação, sendo duas morfológicas. Em nenhuma delas foi evidenciada alteração morfológica, quer fetal ou dos anexos.

A gestação evoluiu sem intercorrências a paciente foi admitida em trabalho de parto, com amniorrexe espontânea e contrações de fraca in- tensidade. No exame admissional apresentava dados vitais normais, sendo constatada dilatação cervical de seis centímetros e saída de quantidade moderada de líquido amniótico claro. Batimentos cardiacos fetais ritmicos, ao redor de 140 por minuto. Colocada sob cardiotocografia contínua, teve boa evolução do trabalho de parto. Quando a dilatação cervical atingiu oito centímetros, a dinâmica uterina intensificou-se e iniciou-se a instalação de analgesia peridural. Repentinamente, o monitor fetal mostrou bradicardia fetal aguda e discreto sangramento por via vaginal. A paciente foi imediatamente submetida a raquianestesia e o feto rapidamente retirado por cesariana, tendo nascido com parada cardiorrespiratória que não respondeu a manobras de reanimação.

O exame dos anexos fetais, feito de forma sumária logo após a dequitação, mostrou inserção velamentosa do funículo umbilical. Os três vasos umbilicais irradiavam-se em leque do coto funicular, terminando todos na margem placentária. Dois deles, os mais longos, estavam integros, e o outro, mais curto, roto em duas partes, uma presa ao coto funicular e a outra à margem placentária (Figura 1). O exame anatomopatológico dos anexos fetais revelou: placenta com $493 \mathrm{~g}$, medindo $17,5 \times 14,0 \mathrm{x}$ $4,5 \mathrm{~cm}$. A face materna apresentava diferenciação em cotilédones. O funículo umbilical media 25,0 $\mathrm{cm}$ de comprimento, estando implantado em situação marginal, notando-se nos $11 \mathrm{~cm}$ proximais à placenta, trifurcação dos vasos e ausência de gelatina de Wharton. O menor dos vasos media $9,0 \mathrm{~cm}$ de comprimento e $0,4 \mathrm{~cm}$ de diâmetro e apresentava-se rompido com hemorragia no foco de laceração, notando-se coto com $4,0 \mathrm{~cm}$ de comprimento, aderido à placenta. Microscopicamente, as estruturas mostravam-se conservadas, com caracteres do terceiro trimestre da gestação. De particular, notavase ausência quase total de eritrócitos nas estruturas vasculares placentárias.

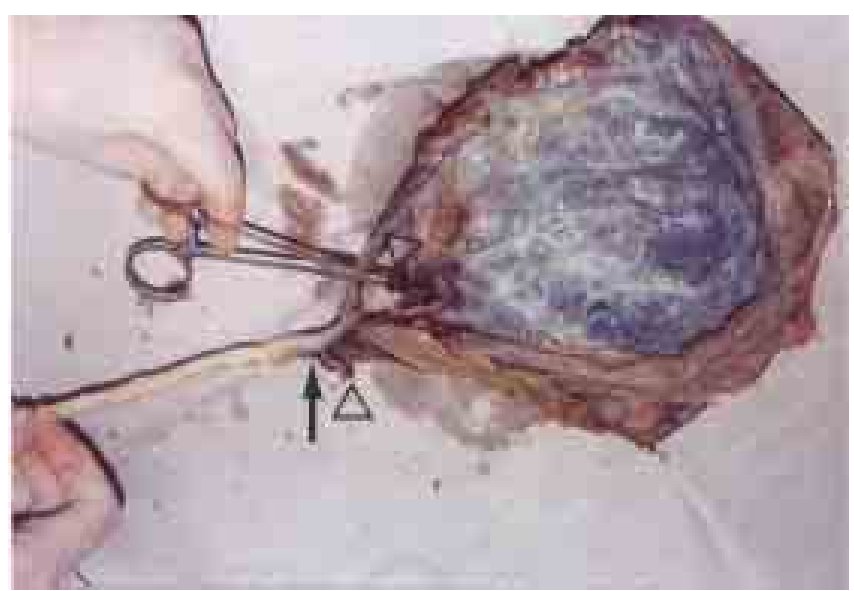

Figura 1 - Placenta e funículo umbilical do caso em tela. A seta negra aponta o local do término da gelatina de Wharton e a separação dos vasos umbilicais. As cabeças de seta mostram as duas extremidades rotas de um dos vasos umbilicais. 


\section{Discussão}

Todos os estudiosos são unânimes ao afirmar que, em função da raridade dos VP, particularmente sua ruptura, embora acompanhada por altas taxas de morbiletalidade fetal, seu possivel diagnóstico é sempre subvalorizado e quase nunca realizado durante a gestação. Para a maioria dos autores existe íntima relação entre a presença de VP e a presença de inserção baixa de placenta, além de outras anomalias placentárias como: placenta bilobada, placenta sucenturiada e placenta com inserção marginal do funículo umbilical $^{1-6}$.

O diagnóstico diferencial dos VP, à luz dos dados clínicos, deve ser feito com: descolamento prematuro de placenta, placenta prévia e, mais raramente, com rotura uterina e rotura de seio marginal placentário. Não cabe neste texto tecer considerações sobre o quadro clínico de cada uma dessas entidades. Todavia, em relação ao descolamento prematuro de placenta e placenta prévia, mais encontradiços, faz parte da conduta médica a amniotomia, que no caso dos VP pode revelar-se medida catastrófica, visto poder provocar a rotura de um dos VP e conseqüente óbito fetal.

Quando da realização dos exames subsidiários (ultra-sonografia e dopplerfluxometria), é necessária atenção para diferenciar VP de outras estruturas que possam simulá-la. Assim, uma separação amniocoriônica pode ser diferenciada de VP pela total ausência de fluxo vascular em seu interior. Do mesmo modo, a presença de procidência ou laterocidência de funículo umbilical poderá sugerir VP. Neste caso, a dopplerfluxometria fará a diferenciação. Outra situação que ultra-sonograficamente poderá simular VP é a presença do seio venoso marginal da placenta; neste caso, também, a dopplerfluxometria fará a diferenciação ${ }^{9}$.

Estudos mais antigos ${ }^{1,2-4}$, utilizando-se de pesquisa de hemoglobina no sangue de origem vaginal, amnioscopia e monitoragem cardiaca fetal, lograram o diagnóstico de VP em número reduzido de casos, com pouca modificação no prognóstico fetal.

Durante as décadas de $1980^{3}$ e de $1990^{4-6}$, o diagnóstico de VP era ainda feito por presunção, baseado em dados clínicos. No final da década de 1980 conseguiu-se o diagnóstico ultra-sonográfico de um único caso de $\mathrm{VP}^{4}$. No final da última década, o diagnóstico de certeza dos VP por ultrasonografia e dopplerfluxometria associadas foi sempre realizado com relativa facilidade ${ }^{7-10}$. À ultra-sonografia, os VP são visualizados como es- truturas lineares ou tubulares próximas ao óstio cervical interno ${ }^{10}$. Na dúvida, evidentemente, a dopplerfluxometria mostrará com clareza a presença de fluxo sangüíneo no interior destas estruturas $^{9}$.

Tendo em vista o exposto, a literatura consultada é unânime ao afirmar que identificados os fatores de risco para VP (inserção baixa de placenta, placentas bilobadas ou sucenturiadas e, notadamente, gemelaridade, mormente se estas condições estiverem associadas a perdas sanguíneas vaginais), obriga-se o tocólogo a perseguir de forma diligente o diagnóstico de VP. Uma vez feito o diagnóstico de certeza, a melhor conduta é aguardar-se a maturidade fetal e submeter a gestante a cesárea. Com esta conduta, adotada em todos os centros onde o diagnóstico pré-natal de VP tornouse rotina, o número de casos diagnosticados evidentemente aumentou, enquanto a nati e neomortalidade neonatal decresceram drasticamente.

Merecem destaque dois trabalhos recentes sobre o tema. O primeiro ${ }^{10}$ mostrou, após estudo prospectivo de 587 gestações, por ultra-sonografia e dopplerfluxometria a cores, ser possivel a visualização da inserção placentária do funículo umbilical em todos os casos, acrescendo apenas 20 segundos no tempo de feitura dos exames. Lograram os autores visualizar dois casos de VP. O segundo trabalho ${ }^{9}$ demonstrou, após estudo com a mesma metodologia, durante 8 anos, que em 93.874 gestações foi possível o diagnóstico de 18 casos de VP, havendo em todos eles a coexistência de alterações morfológicas da placenta e do funículo umbilical.

O caso ora relatado teve como único dado clínico a ressaltar a presença de inserção baixa de placenta, diagnosticada por ultra-sonografia na $18^{\text {a }}$ semana gestacional. Apesar de repetidas ultra-sonografias em várias idades gestacionais e, principalmente, apesar da presença de inserção velamentosa do funículo umbilical, distando 11 centímetros da borda placentária, em nenhum dos exames foi ventilada a possibilidade da existência, quer da inserção velamentosa, quer dos VP. Pela evolução do trabalho de parto podemos presumir que a ruptura de um dos vasos prévios ocorreu no final do período de dilatação, quando a abertura da membrana amniocoriônica o atingiu. A perda sanguínea fetal foi de pequena monta, rápida e fatal, não permitindo que a interrupção rápida da gestação permitisse reanimação cardiorrespiratória do neonato.

Em sintese, diante dos fatores que sabidamente aumentam o risco de VP, como alterações morfológicas placentárias, além de inserção baixa de placenta, gemelaridade e, principal- 
mente, perdas sangüíneas vaginais intermitentes durante a gestação, deve o tocólogo utilizar-se de ultra-sonografia e dopplerfluxometria associadas para o diagnóstico de VP. Embora haja controvérsias, os trabalhos mais recentes preconizam o diagnóstico, preferencialmente, entre a $18^{\mathrm{a}}$ e a $22^{\text {a }}$ semana da gravide $z^{8-10}$. Confirmado o diagnóstico, aguarda-se a maturidade fetal e interrompese a gestação.

\section{SUMMARY}

The differential diagnosis of hemorrhages during the third trimester of pregnancy due exclusively to obstetrical causes includes: abruptio placentae, low insertion of placenta (placenta previa with subtypes), rupture of the uterus, rupture of the marginal placental sinus and rupture of the vasa previa. The three first diagnoses occur more frequently, their epidemiological factors are better known and therefore, have an easier diagnosis. It is common for obstetricians with long practical experience, as well as for ultrasonographers specialized in fetal medicine and who thoroughly know their theory, not to have ever been exposed to practical obstetrical cases of vasa previa or their rupture. The reason to write this article was the fact that, during the past 32 years, we have been working constantly and uninterruptedly in obstetrical practice and we have seen only one case of rupture of vasa previa during labor, which killed the fetus. Initially, we investigated the issue in obstetrical textbooks having no luck whatsoever, except for a couple of lines on the subject. Carrying out our search in depth, we were able to learn that, even though a rarity, today's literature on the subject suggests that it is possible to have a diagnosis for this morbid entity during gestation, and to solve the problem by making the cesarian section mandatory in these cases, leading to a significant reduction in the actual fetal mortality figures which, according to experts, vary between 33 and 100\%.

KEY WORDS: Vasa previa. Velamentous umbilical cord insertion. Fetal death.

\section{Referências}

1. Pent D. Vasa previa. Am J Obstet Gynecol 1979;134:151-5.

2. Carp HJA, Mashiach S, Serr DM. Vasa previa: a major complication and its management. Obstet Gynecol 1979; 53:273-5.

3. Paavonen J, Jouttunpää K, Kangasluoma P, Aro P, Hernonen PK. Velamentous insertion of the umbilical cord and vasa previa. Int $\mathrm{J}$ Gynaecol Obstet 1984; 22:207-11.

4. Gianopoulos J, Carver T, Tomich PG, et al. Diagnosis of vasa previa with ultrasonography. Obstet Gynecol 1987; 69:488-91.

5. Eddleman KA, Lockwood CJ, Berkowitz GS, Lapinski $\mathrm{RH}$, Berkowitz RL. Clinical significance and sonographic diagnosis of velamentous umbilical cord insertion. Am J Perinatol 1992; 9:123-6.

6. Odunsi K, Bullough $\mathrm{CH}$, Henzel J, Polanska A. Evaluation of chemical tests for fetal bleeding from vasa previa. Int J Gynaecol Obstet 1996; 55:207-12.

7. Hertzberg BS, Kliewer MA. Vasa previa: prenatal diagnosis by transperineal sonography with Doppler evaluation. J Clin Ultrasound 1998; 26:405-8.

8. Oyelese KO, Schwarzler P, Coates S, Sanusi FA, Hamid R, Campbell S. A strategy for reducing the mortality rate from vasa previa using transvaginal sonography with color Doppler. Ultrasound Obstet Gynecol 1998; 12:434-8.

9. Lee W, Lee VL, Kirk JS, Sloan CT, Smith RS, Comstock $\mathrm{CH}$. Vasa previa: prenatal diagnosis, natural evolution and clinical outcome. Obstet Gynecol 2000; 95:572-6.

10.Nomiyama M, Toyota Y, Kawano H. Antenatal diagnosis of velamentous umbilical cord insertion and vasa previa with color Doppler imaging. Ultrasound Obstet Gynecol 1998; 12:426-9. 